\title{
Etude comparative sur la méthodologie d'estimation des poids à âges types avant sevrage appliquée aux caprins Créoles producteurs de viande
}

\author{
M. Naves ${ }^{1^{*}}$ A. Menendez Buxadera ${ }^{1}$ \\ G. Alexandre ${ }^{1}$ N. Mandonnet ${ }^{1}$
}

Mots-clés

Caprin Créole - Animal à viande Période de présevrage - Allaitement Contrôle de performances - Croissance - Poids - Guadeloupe.

\begin{abstract}
Résumé
La mise en œuvre d'un programme de sélection chez les ruminants allaitants nécessite un modèle de contrôle de performances pour l'enregistrement des poids et la mesure de la croissance. Un tel schéma a été mis au point en France chez les ovins; en revanche, il n'existe pas de système pour les caprins allaitants. Cette étude examine les possibilités d'application de la méthodologie standard mise au point chez les ovins à viande, dans le cadre de la population rustique de caprins Créoles élevés aux Antilles françaises. Elle s'est appuyée sur la base de données individuelles de croissances accumulées au Domaine expérimental de Gardel en Guadeloupe depuis près de 20 ans, sur un total de 6211 chevreaux jusqu'au sevrage. Différentes méthodes d'estimation des poids à âges types (10, 30 et 70 jours) ont été testées, en comparaison avec la méthode standard développée pour les ovins allaitants en France. Enfin, la variabilité des performances de croissance obtenues a été précisée afin d'établir les premières références d'un contrôle de croissance appliqué aux caprins Créoles. Les conditions d'application et les limites du schéma sont discutées dans le cadre de la population caprine Créole.
\end{abstract}

\section{INTRODUCTION}

Aux Antilles françaises comme dans de nombreux pays tropicaux, l'élevage caprin est orienté vers la production de viande (7). Les animaux sont de race locale rustique appelée Créole $(3,12)$, élevés suivant un système allaitant au pâturage $(1,2)$ (figure 1$)$. Les résultats publiés sur les performances des caprins Créoles $(1,2)$ ont montré le potentiel de ce type d'animal pour la production de viande en zone tropicale, ainsi que les possibilités de sélection de cette race locale pour les aptitudes de croissance en allaitement (10).

Dans ce contexte, il apparaît logique d'examiner les possibilités d'application d'un système de contrôle des performances individuelles comme première étape dans la mise en œuvre d'un programme d'amélioration génétique des caprins Créoles allaitants.

1. Inra, Unité de recherches zootechniques, Domaine Duclos, Prise d'Eau, 97170 Petit Bourg

* Auteur pour la correspondance

Tél. : 0590255933 ; fax : 0590255936 ; email : naves@antilles.inra.fr

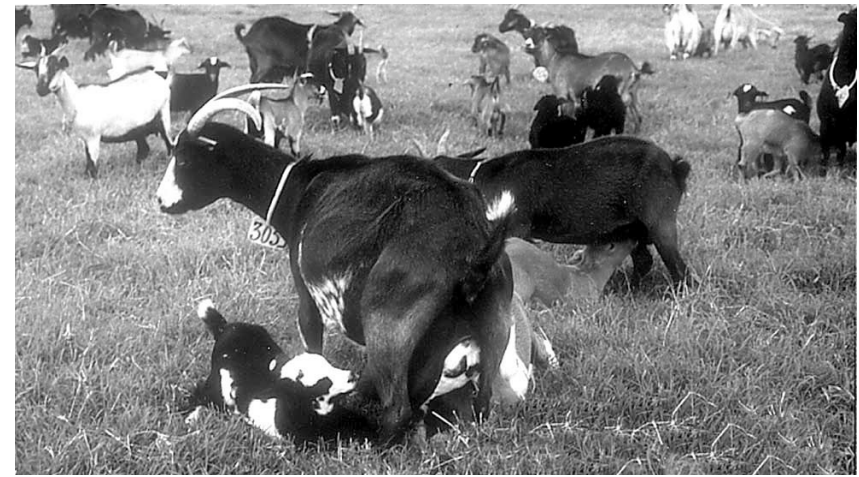

Figure 1 : la chèvre Créole de Guadeloupe est exploitée pour la production de viande suivant un mode allaitant au pâturage.

Malheureusement, il existe peu de références sur les procédures de contrôle de croissance à employer en zone tropicale pour cette espèce. Chez les petits ruminants allaitants, le gain moyen quotidien est le plus souvent estimé de manière globale, par la diffé- 
rence de poids entre la naissance et le sevrage divisée par l'âge au sevrage (5). Dans certains cas, un poids à âge type voisin du sevrage est calculé par interpolation linéaire entre la naissance et le sevrage $(14,17)$ ou par régression linéaire $(15)$. Cependant ces méthodes peuvent donner des résultats biaisés dans la mesure où elles supposent que la croissance est monotone pendant toute la période d'allaitement, ce qui est loin d'être vérifié.

En France, la méthodologie officielle appliquée aux ovins se base sur l'estimation de différents poids vifs pour chaque animal, à l'intérieur de périodes de temps restreintes (8). Les outils développés en France pour le contrôle de performances des ovins à viande $(6,11)$ peuvent constituer un modèle de référence. Ils sont ainsi appliqués dans les programmes d'amélioration génétique des ovins allaitants au Maroc (4) ou en Martinique (13). En revanche, ces procédures n'ont pas été évaluées dans le cas des caprins producteurs de viande, comme les cabris Créoles des Antilles françaises.

La présente étude a eu pour objectif de vérifier la validité de la méthodologie officielle établie pour les ovins producteurs de viande en France dans le cas d'une race locale de caprins producteurs de viande, et d'établir des références sur la variabilité et les bornes des estimations de poids et de croissance présevrage obtenues par l'application de cette méthode officielle aux caprins Créoles de Guadeloupe. Cette adaptation permettrait de bénéficier des outils de contrôle de performances et de gestion des données existant en France, et de l'expérience accumulée dans ce domaine.

\section{MATERIEL ET METHODES}

Le domaine expérimental de Gardel de l'Unité de recherches zootechniques de l'Inra dispose d'une base de données sur les caprins Créoles de Guadeloupe accumulées depuis près de 20 ans dans des conditions d'élevage décrites par Alexandre et coll. $(1,2)$.

Le troupeau de chèvres allaitantes a exploité toute l'année, suivant un système de rotation de pâturages, des prairies de Digitaria decumbens irriguées et fertilisées. De plus, les chèvres ont reçu une complémentation variable suivant leur stade physiologique. La reproduction a été pratiquée par monte naturelle, avec trois périodes de reproduction dans l'année, ce qui a permis d'obtenir un rythme de trois mises bas en deux ans. Le sevrage est intervenu à un âge moyen de $81 \pm 13$ jours et a été effectué par le retrait simultané du troupeau de mères de l'ensemble des chevreaux nés lors d'une période de mises bas. Une prophylaxie régulière a été pratiquée sous forme de détiquage et de vermifugation, afin de contrôler le développement de parasitoses internes et externes chez les jeunes comme chez les adultes. Les pesées de l'ensemble du troupeau ont été réalisées avec une fréquence qui a varié suivant les expérimentations, mais elle a été généralement bimensuelle.

\section{Constitution des fichiers de données analysées}

Les enregistrements individuels des mises bas et des croissances en allaitement entre les années 1980 à 1996 ont été rassemblés. Ils ont concerné un effectif de 7524 animaux nés au total, dont 6403 ont été sevrés ( 85 p. 100), et ont fourni 23836 enregistrements de poids vifs, en plus du poids à la naissance. Seules ont été prises en compte les pesées intervenues au rythme d'une pesée hebdomadaire à une pesée mensuelle. Les animaux ayant présenté un âge au sevrage supérieur à 120 jours ont été éliminés ainsi qu'un groupe d'animaux utilisés dans des expérimentations avec des objectifs vétérinaires. Un fichier dénommé «Total» a ainsi été constitué, comportant 19787 enregistrements de poids vifs en plus du poids à la naissance, pris sur 6211 chevreaux. La figure 2 représente la fréquence des chevreaux en fonction du nombre de pesées enregistrées dans ce fichier : 70 p. 100 des chevreaux ont eu au moins trois pesées enregistrées durant la phase d'allaitement, en plus du poids à la naissance.

La méthode officielle de contrôle de performances en ovins à viande (8) repose sur une succession de quatre pesées à intervalles de $21 \pm 2$ jours à partir de la date de la première naissance dans un troupeau. Pour vérifier cette condition, les données du fichier Total ont été éditées de façon détaillée afin de choisir un échantillonnage de quatre pesées successives séparées au maximum par 23 jours. Seulement 2166 animaux (33,8 p. 100 des individus du fichier Total) ont répondu à cette condition. Ces animaux ont permis de constituer un fichier dénommé « Contrôle ».

\section{Comparaison des méthodes d'estimation des poids à âges types}

Pour chaque animal du fichier Contrôle, quatre méthodes d'estimation des poids à âges types (Pat) (à 10,30, 70 jours) ont été comparées.

\section{Méthode A}

Les poids à âges types sont estimés par interpolation ou extrapolation, suivant la méthode officielle établie par l'Inra et l'Institut de l'élevage pour les ovins viande (8).

L'estimation des poids à âges types est obtenue par interpolation lorsque deux pesées, le poids de naissance non compté, encadrent l'âge type voulu, en appliquant la formule suivante :

$\mathrm{Pat}_{\mathrm{i}}=\mathrm{P}_{\mathrm{n}}+\left[\mathrm{P}_{\mathrm{n}+1}-\mathrm{P}_{\mathrm{n}}\right] /\left[\mathrm{D}_{\mathrm{n}+1}-\mathrm{D}_{\mathrm{n}}\right] *\left[\mathrm{i}-\mathrm{A}_{\mathrm{n}}\right]$

où i est l'âge du chevreau, égal à 10,30 ou 70 jours ; $P_{n}, P_{n+1}, D_{n}$, $\mathrm{D}_{\mathrm{n}+1}$ représentent respectivement les poids et les dates aux visites de contrôle de croissance $n$ et $n+1$; et $A_{n}$ est l'âge à la pesée $n$ $\left(\right.$ avec $\left.A_{n}<\mathrm{i}<A_{n+1}\right)$.

Dans les cas où il ne peut y avoir interpolation, le calcul peut être réalisé par extrapolation inférieure (où $\mathrm{i}<\mathrm{A}_{\mathrm{n}}<\mathrm{A}_{\mathrm{n}+1}$ ) ou extrapolation supérieure (où $A_{n}<A_{n+1}<i$ ). Des normes précises fixées par la méthode officielle (8) définissent les modalités de choix de la méthode et les limites appliquées dans les calculs des Pat ${ }_{i}$ par interpolation ou par extrapolation. Ces conditions ont été utilisées de manière identique dans l'étude sur les poids à âges types des caprins Créoles :

- calcul de Pat ${ }_{10}$ par interpolation, avec un écart maximum entre les pesées $\left(A_{n+1}-A_{n}\right) \leq 23$ jours, ou extrapolation inférieure, avec une limite $\left(A_{n}-i\right) \leq 12$ jours ;

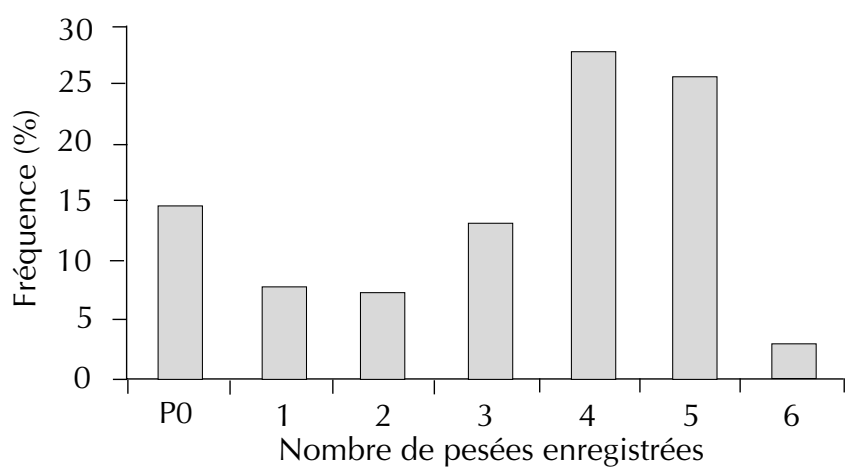

Figure 2 : fréquence des chevreaux Créoles suivant le nombre de pesées enregistrées au Domaine Inra de Gardel (Guadeloupe) ( $n=6211$ chevreaux). P0 représente la classe de chevreaux uniquement présents à la pesée à la naissance. 
- calcul de Pat $_{30}$ par extrapolation supérieure, avec une limite $\left(\mathrm{i}-\mathrm{A}_{\mathrm{n}+1}\right) \leq 10$ jours, ou interpolation, avec un écart maximum entre les pesées $\left(A_{n+1}-A_{n}\right) \leq 44$ jours ;

- calcul de Pat 70 par interpolation, avec un écart maximum entre les pesées $\left(A_{n+1}-A_{n}\right) \leq 23$ jours, ou par extrapolation supérieure, avec une limite $\left(\mathrm{i}-\mathrm{A}_{\mathrm{n}+1}\right) \leq 8$ jours.

\section{Méthode B}

Les Pat à 10, 30, 70 jours d'âge sont estimés selon la même méthode que $\mathrm{A}$, mais avec le poids à la naissance comme résultat de la première pesée.

\section{Méthode C}

Les Pat à 10, 30, 70 jours d'âge sont estimés à partir de l'ajustement, pour chaque animal, d'une équation polynomiale des pesées individuelles en fonction de l'âge, d'un usage habituel dans ce genre d'étude (9). Le modèle initial choisi est une équation de régression linéaire cubique :

Poids $=\mathrm{a}+\mathrm{b}_{1} *$ âge $+\mathrm{b}_{2} *$ âge $^{2}+\mathrm{b}_{3} *$ âge ${ }^{3}$

où $a, b_{1}, b_{2}$ et $b_{3}$ représentent les coefficients de l'équation polynomiale.

A partir de ce modèle, une méthode de régression progressive descendante a été utilisée en appliquant la procédure REG de SAS (16), afin de réduire l'équation polynomiale aux seuls termes significatifs. Cette méthode maintient dans le modèle seulement les termes qui apportent une contribution significative au coefficient de détermination $\left(\mathrm{R}^{2}\right)$ du modèle, déterminée par un test $\mathrm{F}$ de leur part de variance expliquée par rapport à la variance résiduelle. Seuls les termes ayant une valeur de la statistique F significative au seuil de 5 p. 100 ont été maintenus. Sur l'ensemble des chevreaux, le coefficient de détermination du modèle individuel adopté a varié de 0,26 à 1,00 , avec une valeur moyenne de $\mathrm{R}^{2}=0,92$.

\section{Méthode D}

Les Pat à 10, 30, 70 jours d'âge sont estimés à partir d'un ajustement des pesées individuelles au modèle suivant :

$P a_{i}=P_{n}+b_{1} *\left(i-A_{n}\right)+b_{2} *\left(i-A_{n}\right)^{2}+b_{3} *\left(i-A_{n}\right)^{3}$

où $\mathrm{i}$ représente les âges types 10,30 ou 70 jours et $\mathrm{P}_{\mathrm{n}}$ est le poids mesuré à un âge $\mathrm{A}_{\mathrm{n}}$ voisin de l'âge type $\mathrm{i}$ souhaité. La limite de l'intervalle $\left(i-A_{n}\right)$ entre l'âge type $i$ et l'âge à la pesée $A_{n}$ a été fixée à $\pm 12,10$ et 8 jours respectivement pour les calculs des $\mathrm{Pat}_{10}, \mathrm{Pat}_{30}$ et Pat 70 , par analogie avec les bornes utilisées dans la méthode A pour les calculs d'extrapolation.

Les valeurs $b_{1}, b_{2}$ et $b_{3}$ représentent les coefficients d'une régression linéaire cubique, estimée à partir du fichier Total. Pour l'esti- mation de cette régression, chaque valeur de la variable dépendante correspond à la moyenne brute des poids vifs des chevreaux pesés à l'âge $i$, où $i$ varie de 1 à 120 jours. Chaque observation est pondérée par l'inverse de sa variance, pour tenir compte de l'hétérogénéité des moyennes établies avec des nombres de mesures différents. L'équation globale ainsi estimée sur le fichier Total est la suivante :

Poids vif $=16,16+1,058 *$ âge $-0,002711 *$ âge ${ }^{2}-0,00001554 *$ âge $^{3}\left(R^{2}=0,94\right)$.

Les différentes estimations de poids vif obtenues par les quatre méthodes décrites ont été comparées dans une analyse de variance simple (16).

\section{Calculs des paramètres zootechniques de référence pour la population caprine Créole}

La deuxième partie de cette étude a concerné l'analyse de la variabilité des Pat ${ }_{j}$ et des croissances entre ces âges types pour l'établissement de références applicables aux caprins Créoles de Guadeloupe. Il est connu que la variabilité d'un échantillon est d'autant plus représentative de la variabilité de la population que cet échantillon est grand. Cependant, le fichier Contrôle permettant l'application de la méthode officielle A comporte seulement 33,8 p. 100 des animaux de notre base de données. Afin d'augmenter les effectifs, les résultats disponibles sur les autres animaux du fichier Total et obtenus par la méthode $\mathrm{B}$, ou à défaut par la méthode $\mathrm{C}$, donnant les estimations les plus voisines dans la comparaison précédente, ont également été employés. Avec cette nouvelle approche, les Pat ${ }_{\mathrm{i}}$ de 5258 animaux ont été déterminés, soit 85 p. 100 de l'ensemble des chevreaux nés.

Enfin, la croissance à différentes périodes a été calculée à partir de l'estimation de ces Pat ${ }_{1}$ afin de définir des valeurs de références, suivant la méthodologie officielle (8), pour le critère gain moyen quotidien entre 10 et 30 jours d'âge $\left(\mathrm{Gmq}_{10-30}\right)$, qui détermine la valeur laitière de la mère, et pour le critère gain moyen quotidien entre 30 et 70 jours d'âge $\left(\mathrm{Gmq}_{30-70}\right)$, qui renseigne sur la précocité du jeune.

\section{RESULTATS ET DISCUSION}

\section{Comparaison des méthodes d'estimation des poids à âges types}

Les estimations des Pat ${ }_{i}$ par les différentes méthodes sont présentées dans le tableau I. Il est apparu des différences significatives $(\mathrm{P}<0,01)$ entre les méthodes pour $\mathrm{Pat}_{10}$ et $\mathrm{Pat}_{70}$; leurs amplitudes ont cependant été peu marquées. En revanche, $\mathrm{Pat}_{30}$ n'a présenté aucune variation suivant la méthode utilisée (tableau I).

\section{Tableau I}

Moyenne des moindres carrés des poids à âges type ( $\left.\mathrm{Pat}_{\mathrm{i}}\right)$ calculés par différentes méthodes d'estimation, développées dans le texte, sur des chevreaux Créoles de Guadeloupe élevés au Domaine Inra de Gardel en conditions intensives de pâturage durant leur période présevrage ( $n=2166$ chevreaux)

\begin{tabular}{lccc} 
Méthode & Pat $_{\mathbf{1 0}} \mathbf{( h g )}$ & Pat $_{\mathbf{3 0}}$ (hg) & Pat $_{\mathbf{7 0}}$ (hg) \\
\hline A : officielle & $27,9^{\mathrm{a}}$ & $44,7^{\mathrm{a}}$ & $72,9^{\mathrm{a}}$ \\
B : officielle + poids de naissance & $26,6^{\mathrm{c}}$ & $44,9^{\mathrm{a}}$ & $72,3^{\mathrm{ab}}$ \\
C : régression intra animal & $27,1^{\mathrm{b}}$ & $44,9^{\mathrm{a}}$ & $73,7^{\mathrm{a}}$ \\
D : régression à partir de courbe & $26,2^{\mathrm{d}}$ & $44,8^{\mathrm{a}}$ & $71,5^{\mathrm{b}}$ \\
Ecart-type résiduel & 0,190 & 0,263 & 0,472
\end{tabular}

a, b, c et d : les valeurs dans la même colonne présentant des exposants différents diffèrent significativement $(P<0,01)$ 
L'utilisation de la méthode D a fourni une sous-estimation des Pat $_{10}$ et Pat ${ }_{70}$, comparativement aux valeurs obtenues par les méthodes $\mathrm{A}, \mathrm{B}$, et $\mathrm{C}$. Cette observation peut être expliquée par l'inadéquation du modèle polynomial utilisé globalement qui suppose une cinétique identique pour l'ensemble des individus aux différents stades de la croissance. Une description précise de la courbe de croissance des caprins Créoles avant le sevrage nécessiterait une analyse plus approfondie des méthodes d'ajustements à utiliser, par l'application de modèles mixtes mieux appropriés pour décrire l'influence de facteurs de variation à effets fixés ou aléatoires sur des variables longitudinales, comme le poids vif enregistré à différents âges (9).

Une des hypothèses de base de la méthode officielle d'estimation des Pat ${ }_{i}$ est que la croissance est linéaire entre deux pesées successives déterminées dans une courte période. Les résultats de cette étude ont semblé vérifier cette hypothèse de base, puisque les deux méthodes utilisant ce même principe (A et $\mathrm{B}$ ) ont donné des résultats voisins à l'ajustement d'une régression pour chaque animal (C).

Enfin, les méthodes A et B ont abouti à des résultats différents, surtout pour le poids à 10 jours. En effet, l'utilisation du poids à la naissance comme première mesure a abouti à sous-estimer ce premier Pat ${ }_{10}$, du fait de la chute de poids dans les premiers jours après la naissance et du délai nécessaire à l'établissement d'une croissance régulière. La différence entre les résultats obtenus à 70 jours par les méthodes A et B n'a pas été significative et a été liée à l'échantillonnage des pesées utilisées dans les calculs.

La comparaison des méthodes d'estimation des Pat chez les caprins Créoles à viande semble montrer qu'il est possible d'utiliser les modalités de calculs développées pour les moutons à viande en France afin d'estimer ces paramètres avec une bonne précision. La méthode officielle $(\mathrm{A})$ donne les résultats les plus proches d'une régression individuelle $(\mathrm{C})$, méthode plus lourde et nécessitant des moyens adéquats de calculs.

\section{Variabilité des poids à âges types et des croissances correspondantes}

Une combinaison des méthodes A, B et $\mathrm{C}$ a été utilisée pour calculer les poids à âges types et les croissances sur un échantillon le plus représentatif possible, correspondant à 85 p. 100 du fichier Total. Les tableaux II et III présentent les valeurs moyennes et les paramètres de dispersion de chacun de ces caractères de croissance.

Ces résultats ont montré la grande variabilité des différents paramètres, avec, par exemple, des poids à 10 jours qui ont varié de 1 à $5 \mathrm{~kg}$ ou encore des $\mathrm{Gmq}_{10-30}$ de - $43 \mathrm{~g}$ à $160 \mathrm{~g}$, pour des chevreaux élevés en conditions intensives de pâturage. Ces éléments précisent les limites pour la validation des données brutes dans le cadre de l'application d'un contrôle de performances en ferme de caprins Créoles, suivant la méthodologie standard appliquée aux ovins à viande en France (8).

Pour tester la validité de ces Pat ${ }_{\mathrm{i}}$, les auteurs ont recherché parmi les données disponibles les pesées obtenues dans une gamme d'âge de plus ou moins un jour autour des âges types voulus, respectivement de 10, 30 et 70 jours. Les résultats obtenus à partir de ces données sont présentés dans le tableau IV et la figure 3.

Dans ces derniers résultats, aucune donnée n'est en dehors des bornes établies dans l'étape précédente et présentées dans le tableau II. Cette observation semble confirmer, de manière empirique, la validité des bornes établies précédemment. En définitive, il apparaît possible d'utiliser les modalités de calcul et de validation développées pour les moutons à viande comme procédure préliminaire pour les contrôles de performances des caprins Créoles.

\section{Tableau II}

Moyenne et intervalle de variation des poids à âges types des chevreaux Créoles de Guadeloupe élevés au Domaine Inra de Gardel en conditions intensives de pâturage durant leur période présevrage ( $\mathrm{n}=5258$ chevreaux

\begin{tabular}{|c|c|c|c|c|c|}
\hline $\begin{array}{l}\text { Age } \\
\text { (j) }\end{array}$ & $\begin{array}{l}\text { Moyenne } \\
\text { (hg) }\end{array}$ & $\begin{array}{l}\text { Ecart-type } \\
\text { (hg) }\end{array}$ & $\begin{array}{c}\text { Coefficient de variation } \\
(\%)\end{array}$ & $\begin{array}{l}\text { Limite inférieure } \\
\text { (hg) }\end{array}$ & $\begin{array}{l}\text { Limite supérieure } \\
\text { (hg) }\end{array}$ \\
\hline 10 & 27,0 & 5,3 & 20 & 10,0 & 50,0 \\
\hline 30 & 44,9 & 9,2 & 21 & 18,6 & 75,6 \\
\hline 70 & 71,1 & 15,9 & 22 & 30,1 & 130,1 \\
\hline
\end{tabular}

\section{Tableau III}

Moyenne et intervalle de variation des gains moyens quotidiens des chevreaux Créoles de Guadeloupe élevés au Domaine Inra de Gardel en conditions intensives de pâturage durant leur période présevrage ( $n=5258$ chevreaux)

\begin{tabular}{lccccc}
$\begin{array}{l}\text { Classe d'âge } \\
(\mathbf{j})\end{array}$ & $\begin{array}{c}\text { Moyenne } \\
(\mathbf{g} / \mathbf{j})\end{array}$ & $\begin{array}{c}\text { Ecart-type } \\
(\mathbf{g} / \mathbf{j})\end{array}$ & $\begin{array}{c}\text { Coefficient de variation } \\
\mathbf{( \% )}\end{array}$ & $\begin{array}{c}\text { Limite inférieure } \\
(\mathbf{g} / \mathbf{j})\end{array}$ & $\begin{array}{c}\text { Limite supérieure } \\
(\mathbf{g} / \mathbf{j})\end{array}$ \\
\hline $10-30$ & & & 31 & -43 & 160 \\
$30-70$ & 90 & 28 & 30 & 13 & 154 \\
$10-70$ & 74 & 22 & 39 & -23 & 171
\end{tabular}




\section{Tableau IV}

Estimation des poids à âges types (Pat) vrais d'après le poids mesuré à un âge égal à 10, 30 et 70 jours, plus ou moins un jour, chez des chevreaux Créoles de Guadeloupe élevés au Domaine Inra de Gardel

\begin{tabular}{lccccccc} 
Caractère & $\begin{array}{c}\text { Nombre } \\
\text { d'animaux }\end{array}$ & $\begin{array}{c}\text { Age } \\
\text { moyen }\end{array}$ & $\begin{array}{c}\text { Moyenne } \\
\text { (hg) }\end{array}$ & $\begin{array}{c}\text { Ecart-type } \\
\text { (hg) }\end{array}$ & $\begin{array}{c}\text { Coefficient } \\
\text { de variation } \\
\text { (\%) }\end{array}$ & $\begin{array}{c}\text { Limite } \\
\text { inférieure } \\
\text { (hg) }\end{array}$ & $\begin{array}{c}\text { Limite } \\
\text { supérieure } \\
\text { (hg) }\end{array}$ \\
\hline Pat $_{10}$ & 659 & 10,1 & 27,8 & 5,6 & 20 & 13,0 & 43,0 \\
Pat $_{30}$ & 956 & 30,1 & 46,1 & 8,4 & 18 & 24,0 & 70,0 \\
Pat $_{70}$ & 686 & 70,0 & 73,1 & 16,2 & 22 & 36,0 & 114,0
\end{tabular}

Cependant, les résultats présentés dans ce travail sont basés sur les données d'élevage du Domaine expérimental de Gardel, dont le mode de conduite et le système d'alimentation en conditions intensives sont peu appliqués en ferme en Guadeloupe (3). Par ailleurs, les difficultés logistiques pour la collecte des pesées en élevage privé, plus importantes qu'en élevage expérimental, peuvent être source de biais. La régularité des pesées préconisée suivant la
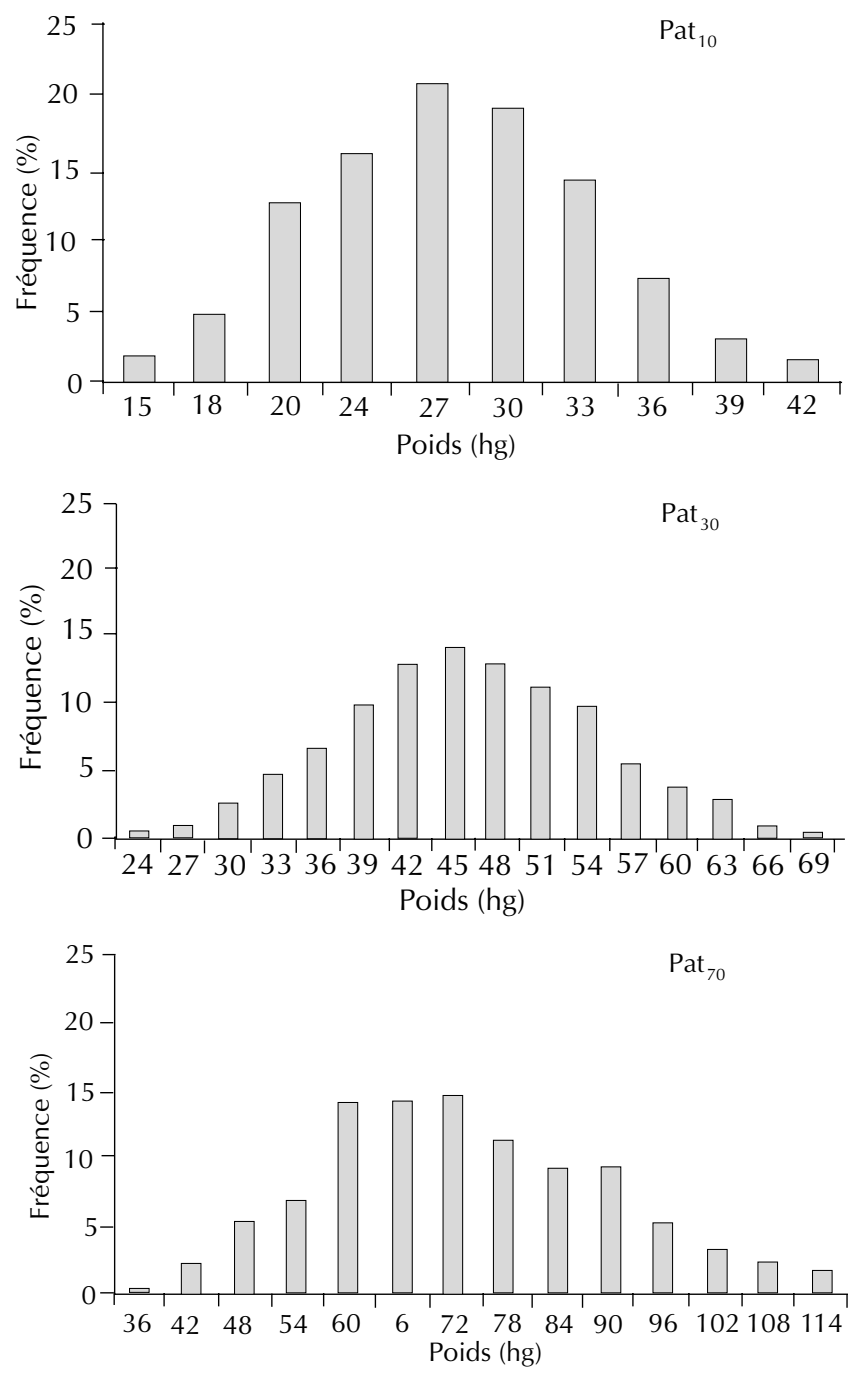

Figure 3 : dispersion des poids à âges types pris à 10 jours $\left(\right.$ Pat $\left._{10}\right), 30$ jours ( Pat $_{30}$ et 70 jours $\left(\right.$ Pat $\left._{70}\right)$ du chevreau Créole élevé en conditions intensives de pâturage au Domaine Inra de Gardel (Guadeloupe). méthode officielle peut aider à réduire les imprécisions. L'extrapolation des résultats au reste de la population caprine de Guadeloupe, suivant des modes de conduite différents, nécessite une étape de validation après un temps d'application dans les conditions d'élevage privé.

Le document de référence (8) précise que l'éleveur doit prévenir l'Etablissement départemental de l'élevage dès la naissance du premier animal (le jour même ou le lendemain). Dans ce cas, la première visite du technicien doit intervenir dans les 21 jours qui suivent la première mise bas. Cette règle doit être respectée pour les caprins Créoles, dont la taille de population ne permet pas de contrôler des effectifs importants. Par ailleurs le système de reproduction appliqué, avec la technique de l'effet mâle, se traduit par la réalisation de 85 p. 100 des mises bas dans les 21 premiers jours de la saison de mise bas. Le non-respect des limites établies et un retard pris lors de la première visite aurait pour conséquence une perte de données. Le tableau $\mathrm{V}$ présente la fréquence cumulée de pertes d'informations pour chaque jour de retard lors des première et deuxième visites. Le taux de pertes est important et il est recommandé de porter une attention particulière à la réalisation de la première visite.

\section{Tableau V}

Fréquence cumulée des pertes de données (pourcentage $d^{\prime}$ individus exclus des calculs de poids à âges types) pour chaque jour de retard pris dans les première et deuxième visites, dans le cadre de la procédure officielle de contrôle de performances

\begin{tabular}{|ccc|} 
Jours de retard & $\begin{array}{c}\text { Première visite } \\
(\%)\end{array}$ & $\begin{array}{c}\text { Deuxième visite } \\
(\%)\end{array}$ \\
\hline 1 & 2 & 1 \\
2 & 4 & 3 \\
3 & 10 & 5 \\
4 & 14 & 7 \\
5 & 18 & 9 \\
6 & 22 & 10 \\
7 & 25 & 11 \\
8 & 28 & 11 \\
9 & 33 & 12 \\
10 & 38 & 12 \\
11 & 44 & 13 \\
12 & 48 & 13 \\
13 & 53 & 14 \\
14 & 58 & 14 \\
\end{tabular}




\section{CONCLUSION}

Les résultats de cette étude montrent qu'il est possible d'initier un système de contrôles de performances des caprins Créoles avec la méthodologie appliquée aux ovins producteurs de viande en France $(6,8)$ et déjà employée aux Antilles françaises pour les ovins Martinik (13). Elle devrait faciliter la réalisation pratique des opérations de contrôle de performances grâce aux outils existants et la gestion des données dans une chaîne de traitement déjà éprouvée.

Par ailleurs, la signification des paramètres employés en relation avec les caractères zootechniques considérés (valeur laitière, précocité) et leur utilisation en vue de l'évaluation de la valeur génétique des caprins Créoles restent à être étudiée de manière plus approfondie. Ainsi, il convient de s'interroger sur l'adéquation aux caprins allaitants en zone tropicale des objectifs et des critères de sélection employés chez les ovins à viande en France (11). En effet, les conditions d'élevage en milieu tropical, où le pâturage prédomine, l'évolution de la courbe de lactation chez les chèvres allaitantes (3) et les différences de développement pondéral dans cette population de petit format sont autant de raisons qui militent pour une révision des critères à employer.

Enfin, en raison de l'absence de références sur la croissance des caprins producteurs de viande en milieu tropical, il apparaît nécessaire de poursuivre l'étude des courbes de croissance de cette espèce et des facteurs de variations influant sur leur évolution. Une perspective intéressante est offerte par les méthodes statistiques d'analyse de modèles mixtes, comme cela a déjà été abordé par Lancelot et coll. (9) pour les ovins du Sénégal.

\section{Remerciements}

Les auteurs remercient J.-P. Poivey pour l'aide apportée à une meilleure compréhension des modalités de calcul des poids à âges types dans le cas des ovins à viande et pour ses conseils sur la présentation des résultats, ainsi que le personnel du Domaine Inra de Gardel pour sa participation à la collecte des données de pesées individuelles. Ils remercient également les correcteurs du comité de rédaction de cette revue pour leurs indications précieuses pour la révision de cet article.

\section{BIBLIOGRAPHIE}

1. ALEXANDRE G., AUMONT G., FLEURY J., COPPRY O., MULCIBA P., NEPOS A., 1997. Production semi-intensive au pâturage de caprins à viande en zone tropicale humide : le cas des cabris Créoles sur pangola (Digitaria decumbens) en Guadeloupe. Inra Prod. Anim., 10 : 43-54.

2. ALEXANDRE G., AUMONT G., FLEURY J., MAINAUD I.C., KANDASSAMY T., 1997. Performances zootechniques de la chèvre Créole de Guadeloupe. Bilan de 20 ans dans un élevage expérimental de I'Inra. Inra Prod. Anim., 10 : 7-20.

3. ALEXANDRE G., AUMONT G., MANDONNET N., FLEURY J., NAVES M., 1999. La chèvre Créole de Guadeloupe (FWI) : une ressource génétique importante pour les Tropiques humides. Bull. Inf. Ressour. génét. Anim., 26 : 45-55.

4. BOUJENANE I., AIT BIHI N., BOUKALLOUCHE A., 1995 Amélioration génétique des ovins au Maroc. In : Gabina D., Ed. Stratégies pour l'amélioration génétique des ovins et caprins. Actes de la réunion du réseau Fao/Ciheam sur les ovins et les caprins, sous-réseau Matériel animal, Sidi-Thabet, Tunisie, 26-28 mars 1995. Saragosse, Espagne, Ciheam, p. 109-119 (Cah. Options Méditerr. 11)

5. BUVANENDRAN V., MAKUSA S.M., CHIRONGA P., 1992. Phenotypic and genetic parameters of weaning traits in Dorper sheep in Zimbabwe. Small Rumin. Res., 7: 369-374.

6. COURNUT J., REHBEN E., 1988. Situation et perspectives des contrôles de performances en ferme appliqués aux bovins allaitants et ovins à viande en France. In : $3^{\mathrm{e}}$ Congrès mondial de reproduction et selection des ovins et bovins à viande, Paris, France, 19-23 juin 1988. Paris, France, Inra, p. 259-273.

7. DEVENDRA C., McLEROY G.B., 1982. Goat production in the tropics. London, UK, CAB, $183 \mathrm{p}$

8. INRA/INSTITUT DE L'ELEVAGE, 1995. Répertoire français des méthodes et procédures de contrôle et d'évaluation génétique des reproducteurs ovins et bovins de races allaitantes. Paris, France, Institut de l'élevage, 105 p. (CR n² 2316, Mai 1995)

9. LANCELOT R., LESNOFF M., TILLARD E., McDERMOTT J.J., 2000. Graphical approaches to support the analysis of linear-multilevel models of lamb preweaning growth in Kolda (Senegal). Prev. vet. Med., 46: 225-247.
10. MANDONNET N., ALEXANDRE G., NAVES M., FLEURY J, AUMONT G., MENENDEZ-BUXADERA A., 1998. Genetic parameters of litter size and preaweaning growth rate of Creole goats of Guadeloupe (FWI). In: 6th World Congress on Genetics Applied to Livestock Production, Armidale, Australia, 11-16 January 1998. Armidale, Australia, 6-WCGALP, p. 165-168.

11. MENISSIER F., BOUIX J., 1992. L'amélioration génétique en France : les contextes et les acteurs - Les bovins et ovins producteurs de viande. Inra Prod. Anim., $\mathrm{n}^{\circ}$ hors-série Eléments de génétique quantitative et applications aux populations animales : 11-23.

12. NAVES M., ALEXANDRE A., LEIMBACHER F., MANDONNET N., MENENDEZ-BUXADERA A., 1998. Avances en los programmas de gestion de los recursos geneticos en los rumiantes del Caribe. In : IV Congreso Iberoamericano de razas autoctonas y criollas, Tampico, Mexico, 22-28 noviembre 1998. Tampico, Mexico, FIRC, p. 78-93.

13. NAVES M., LEIMBACHER F., ALEXANDRE G., MANDONNET N., 2000. Development of animal breeding strategies for the local breeds of ruminants in the French West Indies. In: Galal S., Boyazoglu J., Hammond K., Eds, Workshop on Developing Breeding Strategies for Lower Input Animal Production Environments, Bella, Italy, 22-25 September 1999. Rome, Italy, ICAR, p. 379-385. (Tech. Series No. 3)

14. NOTTER D.R., HOUGH J.D., 1997. Genetic parameter estimates for growth and fleece characteristics in Targhee sheep. J. Anim. Sci., 75: 1729-1737.

15. OLESEN I., SVENDSEN M., KLEMETSDAL G., STEINE T.A., 1995. Application of a multiple-trait animal model for genetic evaluation of maternal and lamb traits in Norwegian sheep. Anim. Sci., 60: 457-469.

16. SAS, 1990. SAS/STAT User's guide, Vers. 6, 4th Ed., Vol. 1 and 2. Cary, NC, USA, SAS Institute, 1848 p.

17. VAN NIEKERK M.M., SCHOEMAN S.J., BOTHA M.E., CASEY N., 1996. Heritability estimates for preweaning growth traits in the Adelaide Boer goat flock. S. Afr. Tydskr. Veek., 26: 6-9.

Reçu le 06.07.2000, accepté le 09.11.2001 


\section{Summary}

Naves M., Menendez Buxadera A., Alexandre G., Mandonnet N. Comparative Study to Estimate Weights at Typical Preweaning Ages of Creole Meat-Type Goats

A model, in which weight and growth performances of suckling ruminants are recorded, is necessary when setting up a breeding program. In France, such a model was developed for sheep, but not for suckling goats. In this study, the standard methodology used for meat-type sheep was applied to the robust Creole goats of French Antilles. Growth data up to weaning of 6211 kids were used; the data had been recorded for nearly 20 years at the experimental farm of Gardel in Guadeloupe. Various methods for weight estimation were tested at the typical ages of 10, 30 and 70 days and compared to the standard method developed for suckling lambs in France. Finally, the variability of growth performances was specified and the first elements to test Creole goat growth were determined. Conditions and limitations of the models applied to Creole goats are discussed.

Key words: Creole goat - Meat animal - Preweaning period Suckling - Performance testing - Growth - Weight - Guadeloupe.

\section{Resumen}

Naves M., Menendez Buxadera A., Alexandre G., Mandonnet N. Estudio comparado sobre la metodología para la estimación de los pesos a edades tipo antes del destete aplicado a los caprinos Creoles productores de carne

La realización de un programa de selección en los rumiantes lactantes necesita de un modelo de control de rendimientos para el registro de los pesos y para la medida del crecimiento. Tal sistema fue establecido en Francia en los ovinos. Sin embargo no existe un sistema para los caprinos lactantes. Este estudio examina las posibilidades de aplicación del método estándar establecido para los ovinos de carne, en el marco de la población rústica de caprinos Creoles criados en las Antillas francesas. Se apoya sobre la base de datos individuales de crecimientos acumulados, del Dominio experimental de Gardel en Guadalupe, desde hace cerca de 20 años y sobre un total de 6211 cabritos, hasta el destete. Se probaron diferentes métodos para estimar los pesos a edades tipo (10, 30 y 70 días) y se compararon con el método estándar desarrollado para los ovinos lactantes en Francia. Finalmente, se precisó la variabilidad de los rendimientos de crecimiento obtenidos, esto con el fin de establecer las primeras referencias de un control de crecimiento aplicado a los caprinos Creoles. Se discuten las condiciones de aplicación y las limitaciones del esquema dentro del marco de la población caprina Creol.

Palabras clave: Caprino Creole - Animal de carne - Periodo predestete - Lanctancia - Prueba de rendimiento - Crecimiento - Peso - Guadalupe. 